\title{
INTELLIGENT VIDEO MONITORING OF THE BEHAVIOR OF RATS IN THE SOCIAL RECOGNITION TEST OF COGNITIVE CAPACITY ON THE WORKING MEMORY
}

\author{
Alexei A. Morozov, Olga S. Sushkova, Yuri V. Obukhov \\ Kotelnikov Institute of Radioengineering and Electronics of RAS, http://www.cplire.ru \\ Moscow 125009, Russian Federation \\ Ilia G. Komoltsev, Margarita R. Novikova, Natalia V. Gulyaeva \\ Institute of Higher Nervous Activity and Neurophysiology of RAS, http://www.ihna.ru \\ Moscow 117485, Russian Federation
}

morozov@cplire.ru, o.sushkova@mail.ru, outaudiofillin@gmail.com, mrnovikova.ihna@mail.ru, nata_ gul@mail.ru, yuvobukhov@mail.ru

Abstract: The aim of this work is automation of neurophysiological experiments, namely, intelligent video monitoring of the behaviour of laboratory rats during the social recognition cognitive test on the working memory. The test is conducted in the box with a sawdust background, simultaneously with EEG recording. Automatic video processing supplies objectivity of the interpretation of the results of the testing and provides new possibilities for the standardizing of the neurophysiological experiments. In the paper, methods and means for the logical description and analysis of the behaviour of experimental animals are considered. Recommendations for improving the experimental technique of the social recognition test are elaborated on the base of the results of the experiments with the automatical video processing.

Keywords: intelligent video monitoring; behaviour analysis; rats; social recognition; object-oriented logic programming

UDC 538.573.61

Bibliography - 15 references

Received - 13.09.2018

RENSIT, 2018, 10(2):269-278

DOI: $10.17725 /$ rensit.2018.10.269

\section{Content}

1. INTRODUCTION (269)

2. Collection of experimental data (271)

3. SEPARATION OF THE FOREgROUND AND BACKGROUND (272)

4. Detection of rats (274)

5. ANALysis of the BEHAVIOR of RATS (275)

6. Conclusion (276)

ReFERENCES (276)

\section{INTRODUCTION}

The test "social recognition" is one of the types of tests for working memory used in neurophysiological studies. The purpose of the test is to test the cognitive capabilities of the experimental animal, namely, its ability to memorize other animals.

During the test, another rat B is placed in the cage (box) with the experimental animal (rat) A (see Fig. 1). Experimental animal A for some time gets acquainted with animal $\mathrm{B}$. This is expressed in the fact that animal A approaches $B$ and sniffs it. These actions can be repeated several times. In the course of the test, the experimenter determines the total time spent by the animal A to get acquainted with the

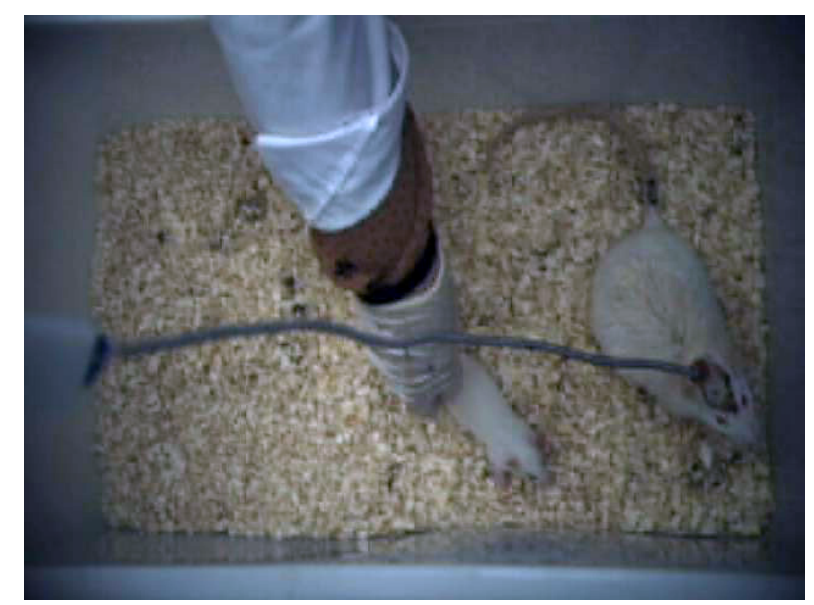

Fig. 1. During the test for social identification in a cage with an experimental animal, a young male is placed. 
animal B. After a few days, the test is repeated again with the same animals and the time spent by the rat $\mathrm{A}$ for acquaintance with the rat $\mathrm{B}$ is determined. If during the re- spent by the rat on acquaintance, is much less than in the first experiment, it is concluded that rat $\mathrm{A}$ remembers rats $\mathrm{B}$, and therefore her cognitive abilities are not violated.

To ensure the correctness of the results of the "social recognition" test, the following test rules are applied:

1. As experimental animals in neurophysiological tests, as a rule, only males are used. Female rats are not used to ensure that the test results are not affected by the menstrual cycles of experimental animals.

2. The animal B is used by young rats (young males). Adult rats are not used so that the test results are not affected by the possible aggression of one animal in relation to another. In addition, due to the fact that animals $A$ and $B$ of the same sex, the possibility of sexual behavior of animals is prevented.

Neurophysiological tests considered in this work are characterized by the following features:

1. Tests were carried out in a plastic box, on the bottom of which sawdust was poured. The litter of sawdust is traditionally used as a hygienic means for the content of experimental animals, including, and for conducting neurophysiological tests. There is an opinion that the sawdust litter is a habitual habitat for the animal, and therefore the absence of sawdust could affect the results of the experiments. Unfortunately, when using automatic methods of analyzing the behavior of an animal, the presence of litter from sawdust becomes a factor seriously complicating the recognition of the animal. The problem is that the animal's hair is about the same color as the sawdust. In addition, the bottom of the cell is unevenly illuminated (usually in the center of the illumination is slightly stronger than at the edges). At the level of image processing algorithms, this leads to the fact that the image of the trunk of the rat and the background image do not have a clear separation in the color spaces "red-green-blue" (RGB) and "huesaturation-brightness" (HSB), and for analyzing video images it is necessary to use more sophisticated (for example, textural) recognition methods.

2. Cognitive tests, considered in the work, are an integral part of the experimental study of the reaction of rats to craniocerebral trauma. During the experiment, an electroencephalogram (EEG) of the animal is recorded, so a cable is connected to the head of the rat, transmitting the EEG signals to the amplifier (see Figure 2). The cable is attached to the head of the rat with a special EEG-cap of a dark brown or bright brown color. The amplifier is a rectangular box suspended above the cage. The movements of the rat on the bottom of the cell lead to a chaotic movement of the EEG cable in the field of view of the video camera. In addition, in the field of view of the camera sometimes falls the case of the amplifier EEG signals. The color of the amplifier's enclosure at certain lighting angles is similar to the color of the coat of experimental animals. Sometimes the body of the amplifier overlaps the image of the rat. All this complicates the analysis of video images of rats and can lead to loss of video capture (see Fig. 2).

Note that the commercial video monitoring systems for neurophysiological experiments (such as Noldus EthoVision XT [1] and Clever Sys SocialScan [2], see review [3]) are not intended for processing video images obtained on the background of sawdust coinciding in color with animal hair. Manufacturers of such systems, as a rule, recommend using special aviaries with a contrasting background (for 


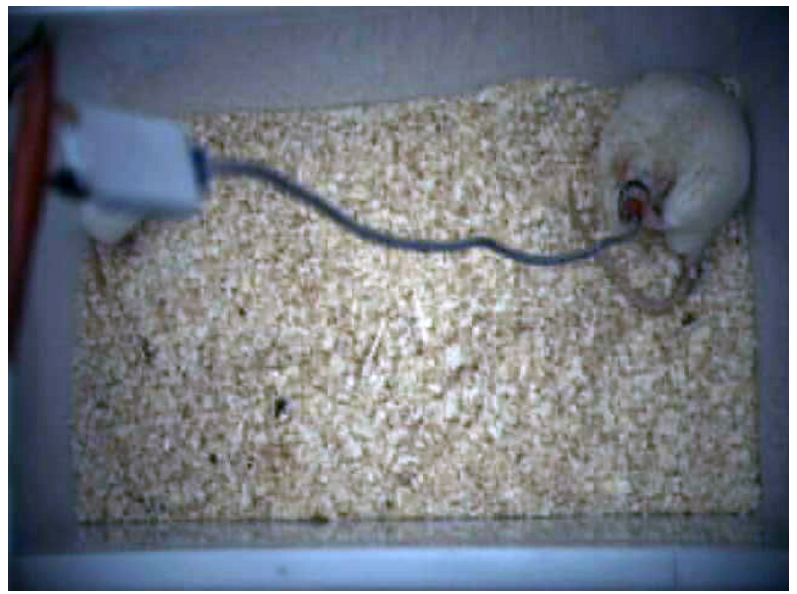

Fig. 2. The EEG cable connecting the head of the rat with the EEG signal amplifier, randomly moves in the field of view of the video camera. The case of the amplifier closed the image of the young male, which resulted in loss of video capture.

example, a black background for white rats or a white background for black rats) for carrying out experiments. It is also recommended to color the experimental animals in colors that are easily discernible against the background of the enclosure. In the course of the work, experiments were performed on the coloration of rats with the help of potassium permanganate (see Fig. 3), but it turned out that this method of coloring makes even more difficult to recognize the shape of the animal against the background of sawdust, since the color of the trunk becomes uneven.

In this paper, the problem of intelligent video monitoring (automatic analysis of video images) of the behavior of rats during

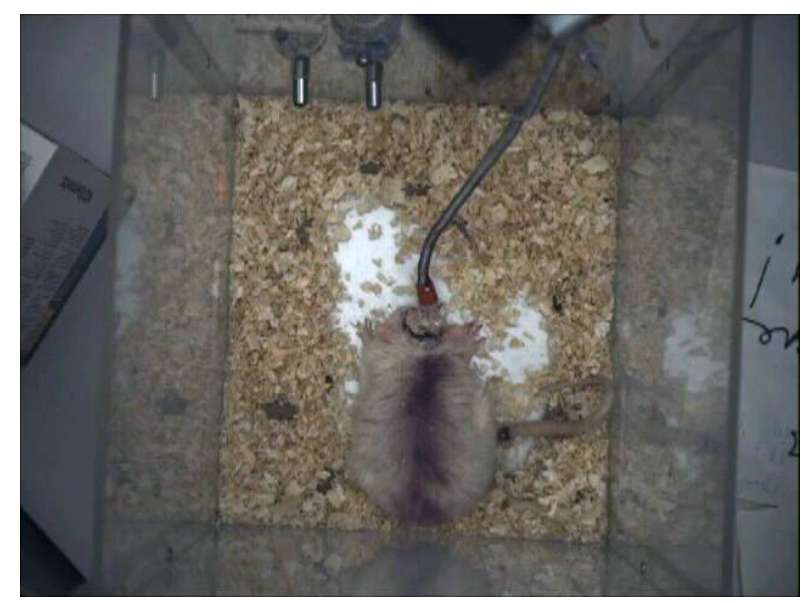

Fig. 3. The contour of the body of the experimental animal is poorly visible against the background of the litter of sawdust. the "social recognition" test, conducted in conjunction with the recording of EEG in boxes with litter of sawdust, is solved. Next, methods and means for the logical description and analysis of the behavior of experimental animals during the test will be examined. In addition, recommendations will be developed to improve the methodology for conducting the "social recognition" test, taking into account bottlenecks and contradictions in the test rules found in the automatic analysis of animal behavior.

\section{COLLECTION OF EXPERIMENTAL DATA}

To carry out the experiments at the Institute of Higher Nervous Activity and Neurophysiology of the Russian Academy of Sciences (IHND and NF RAS), a laboratory stand was installed (see Fig. 4) equipped with high-resolution color cameras DBK 33GX174 (The Imaging Source Europe GmbH, Germany). The video was recorded with a resolution of $640 \times 480$ pixels and a frame rate of $30 \mathrm{~Hz}$ (bitrate 28.79 Mbps, AVI video file format) in order to ensure sufficient detail of video images and at the same time, if possible, reduce the size of video files.

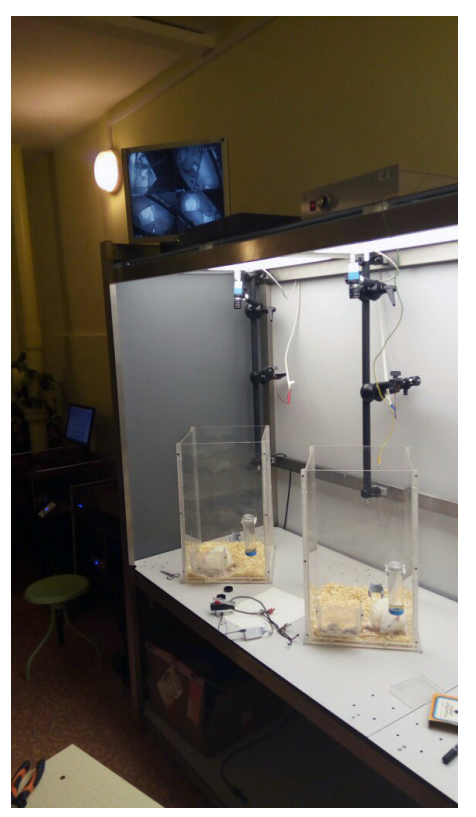

Fig. 4. Laboratory stand in IHND and NF RAS, equipped with color cameras of bigh resolution. 
In accordance with the rules of the "social recognition" test, the following stages of the video image analysis can be distinguished:

1. Recognize the images of two rats $A$ and $B$, where rat $A$ is the animal on which the experiment is performed, and rat $\mathrm{B}$ is the young male planted in the cage during the test.

2. Measure the distance between rats $\mathrm{A}$ and $\mathrm{B}$.

3. Calculate the total time TS, during which rats $\mathrm{A}$ and $\mathrm{B}$ were adjacent to each other, namely, closer to a given threshold of distance SD.

Of course, TS time is only a rough estimate of the time spent by rat $A$ on sniffing of rat B. It will be further shown how adequate this assessment is and what factors are unrecorded.

Let's consider in detail the various stages of analyzing the video image.

\section{SPLIT THE FOREGROUND AND BACKGROUND}

To recognize images of rats, one can use one of the standard algorithms for isolating blobs on a video image, however, the recognition of rats is not limited to the task of selecting blobs, because there is no one-to-one relationship between the analyzed objects (animals) and video image blocks. The exact meaning of the term "blob" depends on which algorithm is used to allocate blobs, so the problem of the relationship between video objects and blobs will be discussed below, after discussing the algorithm for selecting blobs.

Due to the fact that video shooting of rats is carried out against the background of litter from sawdust, it is impossible to apply standard background subtraction algorithms to isolate blobs. When the rat moves along the bottom of the cell, the sawdust is shifted, and the background subtraction algorithms allocate large foreground images, including litter areas, affected in one way or another by the rat as a foreground. In such a situation, a video surveillance system (for example, [1]) can use a background subtraction to study the trail of the rat movement or an approximate evaluation of the rat movement area, but not to recognize the rat body in the image.

The isolation of blobs on the basis of color information and image brightness of the rat also does not allow stable recognition of the rat against the sawdust, as experiments with video processing have shown that images of rats and litter do not have a clear separation in RGB and HSB color spaces [4, 5]. For this reason, texture recognition methods were used to isolate blobs.

Texture method of blob selection uses for the recognition of images of rats the fact that the animal's fur has a smoother surface than sawdust. The main difference of the texture method is that it analyzes the image in a sliding window and therefore, in particular, does not allow to determine the exact coordinates of the recognized elements of the image.

Blobs were allocated by means of the builtin class VideoProcessingMachine [5, 6] of the object-oriented logical language Actor Prolog [7-15]. To improve the quality of recognition, a combination of texture and color methods for selecting blobs was used, namely, the following operations were used to isolate blobs:

1. In the HSB color space, a subspace is distinguished with a saturation from 0 to 100 conventional units (on a scale of 0-255) and brightness from 55 to 255 conventional units (on a scale of 0-255).

2. The modulus of the gradients of the channel "brightness" is calculated as the smoothness of the surface. The calculated values of the modules are normalized; while values in the range 1-10 are reduced to a scale of $0-255$. Then the values are averaged in a sliding window of $27 \times 27$ pixels. After these transformations, pixels with smoothness from 60 to 200 conventional units (on a scale of 0-255) are selected.

3. The selected areas of the image are processed using the "erode" operations by 5 pixels and, then, "dilate" by 5 pixels. 
4. The formed compact fragments of the image are allocated as blobs with the help of the algorithm for quick allocation of blobs TWO_PASS_BLOB_ EXTRACTION, implemented in the class VideoProcessingMachine. The following parameters of the algorithm were set: the borders of the blob (horizontal_blob_ border and vertical_blob_border) - 7 pixels, the minimum admissible area of the blob is 1000 pixels.

5. Of all the blobs detected in the video frame, three blobs with the largest area are selected. Three blobs must be isolated because, in addition to the two rats, a foreign object (the experimenter's hand, the body of the EEG signal amplifier, etc.) can get into the field of view of the video camera, and at this stage of analysis it is impossible to determine which blobs correspond to rats, and which foreign object.

6. Blanks inside blobs are painted over (fill operation).

7. In accordance with the principle of the virtual video processing machine implemented in the VideoProcessingMachine class, the above operations are automatically repeated for each frame of the video image.

8. Based on the information gathered during the processing of the sequence of frames, the trajectories of moving the blobs (the command blb_track_blobs) are computed. The following parameters of the blob trace algorithm were set: the minimum track length (minimal_track_duration) is 25 frames, the maximal allowed invisibility time of the blob (maximal_blob_invisibility_interval) is 5 frames.

9. Choose three blobs with the longest trajectories of displacements. In general, the trajectories in the video frame can be more than three, because new blobs can appear during the analysis of the sequence of frames, and old blobs can be deleted.
Here is the fragment of the program on Actor Prolog that loads the above commands into the virtual machine for video processing: -- Pause video processing: vpm ? suspend_processing, -- Delete all the commands specified previously: vpm ? retract_all_instructions,

-- Reduce the frame size

-- (proportionally, up to a width of 360 pixels): vpm ? img_resize_image $(360,-1)$, -- Create a new foreground mask: vpm ? msk_push_foreground, -- Select pixels with low color saturation: vpm ? msk_select_foreground ('SATURATION', 0, 100), -- Select pixels with high brightness:

vpm ? msk_select_foreground ('BRIGHTNESS', 55,255), -- Calculate the smoothness estimate of the texture: vpm ? pxl_select_image_channel ('BRIGHTNESS'), vpm ? pxl_compute_gradient ('MODULUS'), vpm ? pxl_normalize_pixels $(0,10)$, vpm ? pxl_smooth_image (13),

-- Select pixels with the specified smoothness: vpm ? msk_select_foreground $(60,200)$,

-- Round off the resulting spots:

vpm ? msk_erode_foreground (5), vpm ? msk_dilate_foreground (5),

-- Select blobs:

vpm ? blb_extract_blobs (

'Rats', 'TWO_PASS_BLOB_EXTRACTION'), -- Choose the three largest blobs:

vpm ? blb_select_front_blobs (3, 'FOREGROUND_AREA'),

-- Fill voids in blobs:

vpm ? blb_fill_blobs, -- Trace blobs:

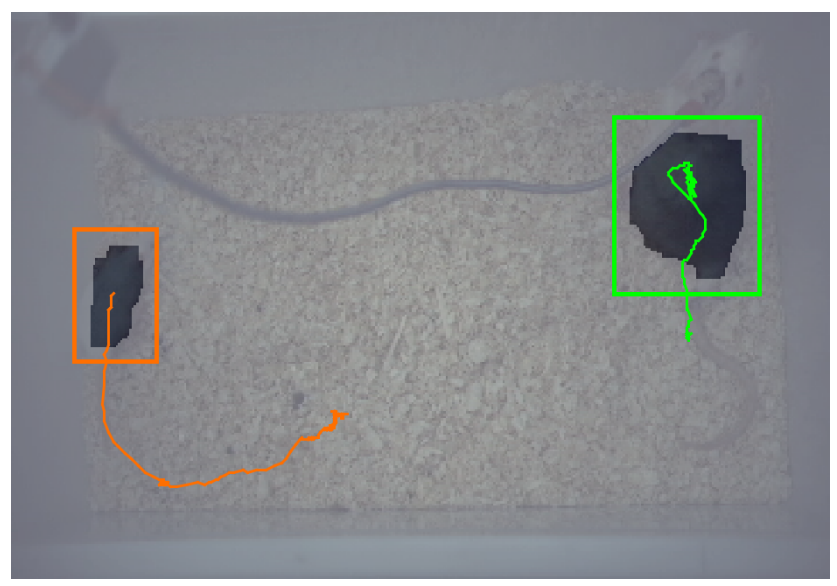

Fig. 5. Blobs selected using the texture method. 
vpm ? blb_track_blobs,

-- Select the three longest tracks:

vpm ? trk_select_front_tracks (

3, 'NUMBER_OF_FRAMES',

'DESCENDING_ORDER'),

-- Select all image channels:

vpm ? pxl_select_image_channel ('ALL'),

-- Resume processing frames:

vpm? process_now.

In Fig. 5 gives an example of the results of video processing. Pixels selected as the foreground image are highlighted in dark gray. Blobs are indicated by green and orange rectangles. In addition, the lines of the corresponding colors show the trajectories of the displacement of the mass centers of the blobs.

The sequence of the video frame processing instructions considered realizes the stage of low-level analysis of the video image $[8,10,11]$. The result of this stage are the graphs describing the trajectories of blob movements in the video frame. At the next stage, the stage of high-level analysis, a logical analysis of these graphs is performed, and conclusions are drawn about the behavior of rats during the test.

\section{DETECTION OF RATS}

In accordance with the rules for carrying out a neurophysiological test, one or two rats may be in a separate video frame. These rats may have several blobs:

1. One blob, if, for example, one rat was selected as a foreground fragment, and the second, for some reason, no, or if the rats are close to each other and merged into one blob.

2. Two blobs, for example, if each rat corresponds to a separate blob.

3. More than two blobs, for example, if the image of the rat was separated by an EEG cable.

In addition, additional blobs corresponding to the EEG-amplifier body, fragments of the EEG-cable, the experimenter's hand, reflections of light from the box walls, and other random factors may appear on the video image. At the level of the low-level analysis of the video image, three blobs of the largest size, with the largest track length, are selected.

The following model of the video scene is used. It is assumed that there are from 0 to 2 rats in the video frame. At first, there are no rats in the video frame, then new rats may appear, but the total number of rats can not exceed two. Each rat has current coordinates in the video frame. If two rats $A$ and $B$ are detected in the video frame, a distance DPAB between the centers of mass of the blobs (in pixels) is used as a rough estimate of the distance between them, and as a more accurate estimate - the distance DMAB between the nearest points on the rectangular borders of the blobs in meters (Actor Prologue allows you to convert the coordinates in pixels to the coordinates in meters in accordance with the specified matrix of projective transformations).

In the course of the experiment, the tracks of the blobs may break off and reappear, and in general it is impossible to determine whether the newly appeared track is the continuation of the trajectory of the rat lost some time ago, or a new object appeared in the frame. In the model, the $\mathrm{DP}$ threshold of the DPAB distance is entered, to which the rat can move for the time that its video capture has been lost. It is assumed that the threshold DT is 75 pixels.

The blobs observed in the video frame are put in correspondence with the alleged images of the rats. The following logical rules apply:

1. When analyzing the next video frame, the logic program tries to place the observed blob tracks in correspondence with the rats detected during the processing of the previous frames. In accordance with each $\mathrm{BV}$ block, the rat is located closest, however, no further than the preset threshold DT.

2. If no rat is located near the DT to the BV block and the number of recognized rats is less than two, it is considered that the BV blob corresponds to the new object. In this case, a new rat is added to the model, and coordinates of the BV blob are taken as its coordinates. 
3. If there are rat RIs in the model that do not have any blobs in the current frame and in the previous frames there were BI blobs whose tracks disappeared in the current frame, the program assumes that some rats turned out to be invisible in the current frame (for example, their image was blocked by an extraneous object). In this case, each rat RI is matched with the nearest BI blob if the distance between them does not exceed the threshold DT.

4. If there was an RI rat in the model, in accordance with which no blobs were delivered, but in the previous frame, the DMAB distance between the two rats did not exceed the SD threshold (taken equal to 0.02 meters), it is considered that the rats came close to each other, and their images merged. The coordinates of the rat RI are taken equal to the coordinates of the rat currently visible.

The experiments showed that the above set of rules allows to adequately interpret the video image of rats, including in situations where rats are approaching and moving away from each other. In this situation, the model of the video scene used does not distinguish rat $\mathrm{A}$ from rat $\mathrm{B}$, and this is its drawback. However, this is unimportant from the point of view of the test results, since the experimenter is only interested

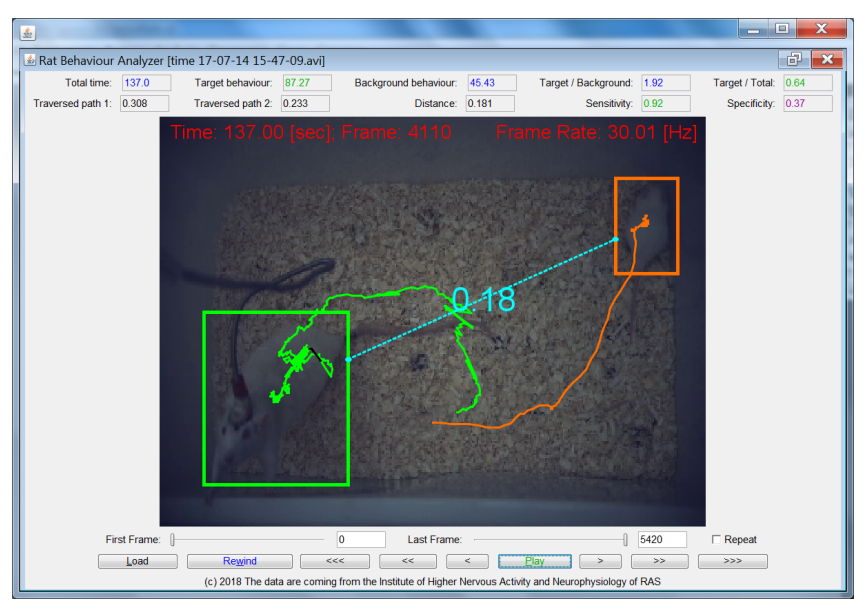

Fig. 6. The logic program analyzes the behavior of rats. The blue line indicates the distance between the rats. The remaining notations are the same as in Fig. 5.
Table 1.

Results of the analysis of the behavior of rats.

\begin{tabular}{|c|c|c|c|}
\hline № & Test time (s) & $\begin{array}{c}\text { Share of social } \\
\text { activity (sec) }\end{array}$ & $\begin{array}{c}\text { Social activity } \\
(\%)\end{array}$ \\
\hline 1 & 181 & 15 & 8 \\
\hline 2 & 181 & 112 & 62 \\
\hline 3 & 181 & 120 & 67 \\
\hline 4 & 181 & 133 & 74 \\
\hline 5 & 181 & 127 & 70 \\
\hline 6 & 181 & 140 & 77 \\
\hline 7 & 181 & 109 & 60 \\
\hline 8 & 181 & 126 & 69 \\
\hline 9 & 185 & 180 & 97 \\
\hline
\end{tabular}

in the distance between the rats. Conditionally it is considered that the rat A corresponds to blobs, the size of which was the largest compared to other blobs at the time of its appearance. Rat A is marked green, and rat B is orange (see Fig. 6).

\section{ANALYSIS OF THE BEHAVIOR OF RATS}

Based on the rules of the analysis of the video scene, formulated in the previous section, a logical program was developed that analyzes the behavior of rats during a neurophysiological test. 9 experimental video recordings were analyzed (see Table 1). In the algorithm for analyzing the behavior of rats, it was assumed that rat A sniffed rat $B$ when the observed distance between DMAB rats was $\leq 0.02$ meters.

To assess the quality of recognition, all 9 videos were analyzed manually. Time intervals have been identified in which rat A sniffs rat B. Table 2 gives estimates of the sensitivity and

Table 2.

Estimates of the sensitivity and specificity of the recognition algorithm.

\begin{tabular}{|c|c|c|}
\hline № & Sensitivity & Specificity \\
\hline 1 & 0.88 & 1.0 \\
\hline 2 & 1.0 & 0.63 \\
\hline 3 & 0.93 & 0.35 \\
\hline 4 & 0.95 & 0.38 \\
\hline 5 & 0.95 & 0.43 \\
\hline 6 & 0.98 & 0.4 \\
\hline 7 & 0.93 & 0.37 \\
\hline 8 & 0.96 & 0.56 \\
\hline 9 & 1.0 & 0.08 \\
\hline
\end{tabular}


specificity of the algorithm for recognizing the desired behavior of a rat, calculated by comparing the results of manual marking of video recordings with the results of automatic analysis.

The estimates in Table 2 show that the logical method of recognition has a sufficiently high sensitivity (in most cases, above 90\%), but the specificity of the method is rather low (in most cases, less than $50 \%$ ). A detailed analysis of video records showed that the recognition algorithm is mistaken in the following cases:

Rats can be near, but do not sniff each other. For example, in Fig. 7b, the rats practically touch each other, but their attention is directed to an object from above.

Rat B can also sniff Rat A, and the algorithm will erroneously detect the social activity of rat A (see the example in Fig. 7c).

There were reported cases of abnormal behavior of rat A when it captured rat B with forelegs and for a long time sniffed the genitalia of rat B (see the example in Fig. 7d). Most likely, these actions were not a manifestation of the sexual behavior of rat $A$, because she did not attempt to perform coitus. Perhaps these actions are a manifestation of the deto-oriented

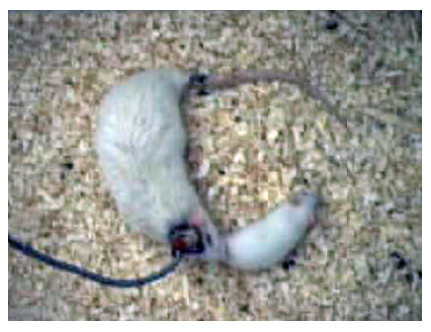

(a)

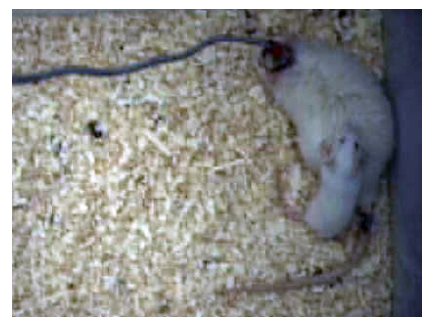

(c)

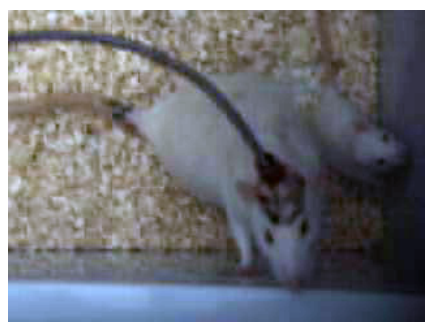

(b)

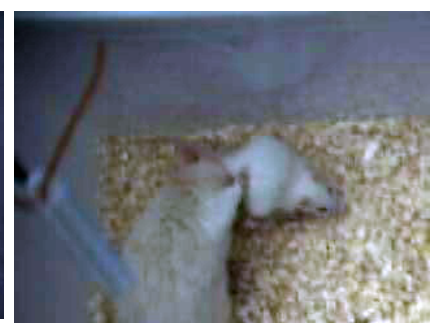

(d)
Fig. 7. Examples of behavior of rats: (a) Rat $A$ sniffs rat $B$, (b) Rats just sit next to, (c) Rat B sniffs rat $A$, (d) Quasi-sexual behavior of rat $A$. behavior of an adult in relation to a young rat. These actions are also erroneously interpreted by the algorithm as recognition by rat A of rat B.

With the manual analysis of the results of the "social recognition" test, the above examples of the behavior of rats can be interpreted in different ways, depending on the experimenter's opinion, which, of course, casts doubt on the objectivity of the conducted study of the behavior of rats. Low estimates of the specificity of automatic recognition of the desired behavior of rats indicate that to ensure the correctness of test results, it is necessary to make changes in the methodology for conducting it. Based on the experiments performed, the following additional test rules can be recommended:

The test must be carried out in a large enough enclosure to reduce the chance of the rats being accidentally located next to each other (without communication between the rats).

At the beginning of the test, the young male B must be inserted sufficiently far from the rat A so that the automatic recognition algorithm has time to carry out the video capture of both rats separately.

It is advisable to investigate the possibility of decreasing the probability of the deto-oriented behavior of the rat A during the test.

It is advisable in some way to restrict the freedom of movement of rat B in an aviary to reduce the likelihood of an erroneous recognition of the situation of "rat $B$ sniffing rat $A "$ as a manifestation of social activity on the part of rat $A$.

\section{CONCLUSION}

The method and software for intelligent video monitoring of laboratory rats on the basis of the object-oriented logical language Actor Prolog [15] was developed. The method allows to analyze video images of experimental rats in non-standard conditions when it is impossible to apply existing systems of animal behavior analysis. In particular, it allows analyzing the 
social behavior of rats in a box with a bed of sawdust under conditions of low contrast of images and uneven illumination. A logical description and analysis of the behavior of rats with a cognitive test on the working memory of "social identification" was carried out. Based on the results of the experiments, recommendations were developed for improving the test procedure.

\section{AKNOLEJMENT}

The authors are grateful to Anna Olegovna Manolova and Irina Pavlouna Levshina (IHND and NF RAS) for their help in conducting the experiments.

The study was carried out at the expense of a grant from the Russian Science Foundation (project No. 16-11-10258).

\section{REFERENCES}

1. Noldus LP, Spink AJ, Tegelenbosch RA. EthoVision: a versatile video tracking system for automation of behavioral experiments. Behavior Research Methods, 2001, 33(3):398-414.

2. Clever Sys Inc; 2018. Available from: http:// cleversysinc.com.

3. Tscharke M, Banhazi TM. A brief review of the application of the machine in livestock behaviour analysis. Agrárinformatika. Journal of Agricultural Informatics, 2016, 7(1):23-42.

4. Morozov AA, Sushkova OS. On the development of methods and algorithms based on object-oriented logical programming for video monitoring of laboratory rats. In: Sat. Works of the IV International Conference and the Youth School "Information Technologies and Nanotechnologies" (ITNT-2018), April 2427, 2018, Samara, Samara National Research University, 2018, p. 1182-1192.

5. Morozov AA, Sushkova OS, Vaniya SM. Development of Methods and Algorithms Based on Object-Oriented Logic Programming for Video Monitoring of Laboratory Rodents. In: Signal-Image Technology \& Internet-Based Systems (SITIS-2017), 13th
International Conference IEEE, 2017, p. 311-318.

6. Morozov AA, Sushkova OS. Virtual machine for low-level processing of video images in Actor Prolog. In: Sat. Works of the IV International Conference and the Youth School "Information Technologies and Nanotechnologies" (ITNT-2018), April 24-27, 2018, Samara, Samara National Research University; 2018, p. 1275-1285.

7. Morozov AA, Sushkova OS, Polupanov AF. Object-Oriented Logic Programming of Intelligent Visual Surveillance for Human Anomalous Behavior Detection. In: RivasLopez M, Sergiyenko O, Flores-Fuentes W, Rodriguez-Quinonez JC (eds.). Optoelectronics in Machine Vision-Based Theories and Applications. Hershey, IGI Global Publications, 2019, p. 134-187.

8. Morozov AA, Sushkova OS. Real-time analysis of video by means of the Actor Prolog language. Computer Optics, 2016, 40(6):947-957.

9. Morozov AA, Sushkova OS, Polupanov AF. Towards the Distributed Logic Programming of Intelligent VisualSurveillanceApplications. In: Pichardo-Lagunas O, Miranda-Jimenez $\mathrm{S}$ (eds.). Advances in Soft Computing, Part II. Cham: Springer International Publishing, 2017, p. 42-53.

10. Morozov AA. Development of a Method for Intelligent Video Monitoring of Abnormal Behavior of People Based on Parallel Object-Oriented Logic Programming. Pattern Recognition and Image Analysis, 2015, 25(3):481-492.

11. Morozov AA, Vaish A, Polupanov AF, Antciperov VE, Lychkov II, Alfimtsev AN, et al. Development of the concurrent objectoriented logic programming platform for the intelligent monitoring of anomalous human activities. In: Plantier G, Schultz T, Fred A, Gamboa H (eds.). BIOSTEC 2014, vol. 511 of CCIS. Heidelberg, Springer, 2015, p. 82-97. 
12. Morozov AA. Logic Object-Oriented Model of Asynchronous Concurrent Computations. Pattern Recognition and Image Analysis, 2003, 13(4):640-649.

13. Morozov AA. Actor Prolog: an ObjectOriented Language with the Classical Declarative Semantics. In: Sagonas K, Tarau P (eds.). IDL 1999. Paris, France, 1999, p. 39-53.

14. Morozov AA. The Prolog with Actors. Programmirovanie, 1994, (5):66-78 (in Russ.).

15. Morozov AA, Sushkova OS. The Intelligent Visual Surveillance Logic Programming Web Site, 2018. Available from: http://www. fullvision.ru. 